International Journal of Bifurcation and Chaos

(c) World Scientific Publishing Company

\title{
IMPROVED HOMOCLINIC PREDICTOR FOR BOGDANOV-TAKENS BIFURCATION
}

\author{
YU.A. KUZNETSOV, H.G.E. MEIJER \\ Department of Applied Mathematics, University of Twente, Zilverling Building, P.O. Box 217, 7500AE \\ Enschede, The Netherlands \\ [I.A.Kouznetsov, H.G.E.Meijer]@utwente.nl \\ B. AL HDAIBAT, W. GOVAERTS \\ Department of Applied Mathematics and Computer Science, Ghent University, Krijgslaan 281-S9, 9000 \\ Gent, Belgium \\ [Bashir.AlHdaibat, Willy.Govaerts]@UGent.be \\ Received (to be inserted by publisher)
}

\begin{abstract}
An improved homoclinic predictor at a generic codim 2 Bogdanov-Takens (BT) bifucation is derived. We use the classical "blow-up" technique to reduce the canonical smooth normal form near a generic BT bifurcation to a perturbed Hamiltonian system. With a simple perturbation method, we derive explicit first- and second-order corrections of the unperturbed homoclinic orbit and parameter value. To obtain the normal form on the center manifold, we apply the standard parameter-dependent center manifold reduction combined with the normalization, that is based on the Fredholm solvability of the homological equation. By systematically solving all linear systems appearing from the homological equation, we remove an ambiguity in the parameter transformation existing in the literature. The actual implementation of the improved predictor in MatCont and numerical examples illustrating its efficiency are discussed.
\end{abstract}

Keywords: Normal form, Bogdanov-Takens bifurcation, homoclinic orbit, center manifold, MatCont

\section{Introduction}

Equilibria with two zero eigenvalues can appear in generic smooth families of autonomous ODEs

$$
\dot{x}=f(x, \alpha), \quad x \in \mathbb{R}^{n}, \alpha \in \mathbb{R}^{m},
$$

when $n \geq 2$ and $m \geq 2$. If the Jordan block

$$
\left(\begin{array}{ll}
0 & 1 \\
0 & 0
\end{array}\right)
$$

is associated to these eigenvalues, such event is called a Bogdanov-Takens (BT) bifurcation. Bogdanov-Takens bifurcations of different codimensions play an important role in the analysis of dynamics of (1), since they imply the appearance of homoclinic orbits to saddle equilibria near the critical parameter values. These local homoclinic orbits are located in the 2D invariant center manifolds.

The exact bifurcation scenario near a BT-point is determined by an unfolding of the critical ODE on the $2 \mathrm{D}$ center manifold, with as many unfolding parameters as the codimension of the bifurcation. More 
precisely, the bifurcation diagram of the unfolding depends on the coefficients of the critical normal form on the center manifold. The restriction of (1) to any center manifold at the critical parameter values can be transformed by formal smooth coordinate changes to the form

$$
\left\{\begin{array}{l}
\dot{w}_{0}=w_{1}, \\
\dot{w}_{1}=\sum_{k \geq 2}\left(a_{k} w_{0}^{k}+b_{k} w_{0}^{k-1} w_{1}\right)
\end{array}\right.
$$

(see, for example, [Arnold, 1983; Guckenheimer \& Holmes, 1983]).

One of the most frequently used in applications bifurcations is the codim 2 BT with $a_{2} b_{2} \neq 0$, for which the complete bifurcation diagram for a generic two-parameter unfolding was obtained in 1970s [Takens, 1974; Bogdanov, 1975, 1976b,a]. The bifurcation diagram is presented in many textbooks (e.g., [Guckenheimer \& Holmes, 1983; Kuznetsov, 2004]) and includes three bifurcation curves at which fold, Andronov-Hopf, and saddle-homoclinic bifurcations occur. The existence of the latter bifurcation curve makes this bifurcation particularly interesting, since it can be detected by the linear (eigenvalue) analysis but predicts a global (homoclinic) bifurcation for nearby parameter values.

There are standard methods to continue fold (limit point) and Hopf bifurcations of equilibria of ODEs in two parameters [Govaerts, 2000], as well as techniques for the two-parameter continuation of saddle homoclinic orbits given a suitable initial point [Champneys et al., 1996; De Witte et al., 2012]. One needs, therefore, a special predictor method to initialize the continuation of homoclinic solutions from a BT point. Since the homoclinic orbit shrinks to the equilibrium while tracing the homoclinic bifurcation curve, this predictor could be based on asymptotics for the bifurcation parameter values and the corresponding small homoclinic solutions in the phase space. Such asymptotics, i.e. a homoclinic predictor, were first derived by Beyn [1994]. Due to the existence of the parameter-dependent 2D center manifold near the bifurcation, the problem splits naturally into two sub-problems: (a) derive the asymptotics for the canonical normal form on the 2D center manifold; (b) transform the approximate homoclinic orbit into the phase- and parameter-space of a given generic $n$-dimensional ODE (1).

The general first step in solving sub-problem (a) is to perform a singular rescaling that brings the 2D normal form into a Hamiltonian system (with an explicit homoclinic solution) plus a small perturbation. Then one can use several methods to obtain an asymptotic expression for the parameter values corresponding to the perturbed homoclinic orbit. One possibility is to apply the classical PontrayaginMelnikov technique [Guckenheimer \& Holmes, 1983] or equivalent branching methods [Beyn, 1994]. This technique easily provides the first-order approximation for the bifurcation parameter values in the perturbed Hamiltonian system, as well the zero-order approximation for the homoclinic orbit (i.e. the unperturbed Hamiltonian homoclinic loop). Obtaining higher-order approximations for the homoclinic solution with any of these methods is more involved and - according to our best knowledge - was never attempted before. The sub-problem (b) can either be solved with a Lyapunov-Schmidt method [Beyn, 1994], or using a parameter-dependent center manifold reduction combined with the normalization and based on the Fredholm solvability applied to the homological equation ([Beyn et al., 2002] and below). However, all published solutions to this sub-problem are either incomplete or contain errors.

In the present paper, we revisit both sub-problems (in reversed order). For the sub-problem (a), we use the classical perturbation technique that allows us to derive a quadratic approximation to the homoclinic bifurcation curve and to obtain explicit first- and second-order corrections of the unperturbed homoclinic orbit. These corrections exhibit a counterintuitive behavior that will also be discussed. For the sub-problem (b), we systematically solve all linear systems appearing from the homological equation, thus removing an ambiguity in the parameter transformation present in [Beyn et al., 2002]. By collecting all these results, we formulate an improved homoclinic predictor at a generic codim 2 BT bifucation. This new predictor is implemented in MatCont [Dhooge et al., 2003, 2008] and proved to be more robust than the previous one based on [Beyn et al., 2002].

The paper is organized as follows. In section 2, we describe the parameter-dependent center manifold reduction with normalization, which is an improved version of the method introduced in [Beyn et al., 2002]. In section 3, we reduce the normal form to a perturbed Hamiltonian system and explicitly derive the firstand second-order approximations for the homoclinic solution. Section 4 combines the results obtained in the 
previous sections and provides the explicit computational formulas for the improved homoclinic predictor. We present several numerical examples using an implementation in MatCont in Section 5.

\section{Center manifold reduction combined with normalization}

The predictor for homoclinic orbits emanating from equilibrium bifurcations is constructed within the framework of parameter-dependent center manifold reduction combined with normalization [Beyn et al., 2002], that we briefly recall now.

\subsection{Method}

Suppose that system (1) has a codim 2 equilibrium $x=0$ at $\alpha=0$ and consider a normal form on the center manifold for this bifurcation

$$
\dot{w}=G(w, \beta), \quad G: \mathbb{R}^{n_{c}+2} \rightarrow \mathbb{R}^{n_{c}} .
$$

Here $w \in \mathbb{R}^{n_{c}}$ parametrizes the $n_{c}$-dimensional parameter-dependent center manifold, while $\beta \in \mathbb{R}^{2}$ are the unfolding parameters. For all five codimension-2 equilibrium bifurcations these normal forms are well known (see, for example [Kuznetsov, 2004]). Suppose that an exact or approximate formula is available that gives an emanating codimension-1 bifurcation branch for the normal form (3). In order to transfer this to the original equation (1) we need a relation

$$
\alpha=K(\beta), \quad K: \mathbb{R}^{2} \rightarrow \mathbb{R}^{2}
$$

between the unfolding parameters $\beta$ and the original system parameters $\alpha$ and, moreover, we need a center manifold parametrization

$$
x=H(w, \beta), \quad H: \mathbb{R}^{n_{c}+2} \rightarrow \mathbb{R}^{n} .
$$

Taking (4) and (5) together as $(x, \alpha)=(H(w, \beta), K(\beta))$ yields the center manifold for the suspended system $\dot{x}=f(x, \alpha), \dot{\alpha}=0$. The invariance of the center manifold implies the homological equation

$$
H_{w}(w, \beta) G(w, \beta)=f(H(w, \beta), K(\beta)),
$$

which we can solve using Taylor series by a recursive procedure based on Fredholm's solvability condition that will give the Taylor coefficients with multi-index $\nu$ of $G$ and $H$ with respect to $w$ and $\beta$. We write the Taylor expansion for $f$ at $\left(x_{0}, \alpha_{0}\right)=(0,0)$ as

$$
\begin{aligned}
f(x, \alpha) & =A x+\frac{1}{2} B(x, x) \\
& +J_{1} \alpha+A_{1}(x, \alpha)+\frac{1}{2} J_{2}(\alpha, \alpha) \\
& +\mathcal{O}\left(\|x\|^{3}+\|x\|\|\alpha\|^{2}+\|x\|^{2}\|\alpha\|+\|\alpha\|^{3}\right),
\end{aligned}
$$

where $A=f_{x}\left(x_{0}, \alpha_{0}\right), J_{1}=f_{\alpha}\left(x_{0}, \alpha_{0}\right)$, and $B, A_{1}$, and $J_{2}$ are the standard multilinear forms.

For $\nu=0$ this procedure gives the critical normal form coefficients, while the coefficients with $|\nu| \geq 1$ yield the necessary data on the parameter dependence.

\subsection{Reduction near the BT-bifurcation}

Let $x_{0}=0, \alpha_{0}=0$ be a BT-point. Let $q_{0}$ and $q_{1}$ be real, linearly independent generalized eigenvectors of the Jacobian matrix $A=f_{x}\left(x_{0}, \alpha_{0}\right)$,

$$
A q_{0}=0, \quad A q_{1}=q_{0}
$$

and $p_{0}$ and $p_{1}$ be the corresponding eigenvectors of the transposed matrix $A^{T}$

$$
A^{T} p_{1}=0, \quad A^{T} p_{0}=p_{1} .
$$

Using the standard inner product $\langle$,$\rangle we can assume that the vectors satisfy$

$$
\begin{aligned}
& \left\langle q_{0}, p_{0}\right\rangle=\left\langle q_{1}, p_{1}\right\rangle=1, \\
& \left\langle q_{1}, p_{0}\right\rangle=\left\langle q_{0}, p_{1}\right\rangle=0 .
\end{aligned}
$$


The smooth normal form for the BT bifurcation introduced in [Guckenheimer \& Holmes, 1983] is

$$
\begin{aligned}
\dot{w}=G(w, \beta) & =\left(\begin{array}{c}
w_{1} \\
\beta_{1}+\beta_{2} w_{1}+a w_{0}^{2}+b w_{0} w_{1}
\end{array}\right) \\
& +\mathcal{O}\left(\|w\|^{3}+\|\beta\|\|w\|^{2}\right) .
\end{aligned}
$$

This normal form is slightly different but equivalent to that used in [Bogdanov, 1976b,a], where the second unfolding term was $\beta_{2} w_{0}$.

We use the homological equation (6) with the expansions

$$
\begin{aligned}
K(\beta) & =K_{1} \beta+\frac{1}{2} K_{2} \beta_{2}^{2} \\
& +\mathcal{O}\left(\beta_{1}^{2}+\left|\beta_{1} \beta_{2}\right|+\left|\beta_{2}\right|^{3}\right) \\
H(w, \beta) & =H_{01} \beta+\left[q_{0}, q_{1}\right] w+\frac{1}{2} H_{20,0} w_{0}^{2} \\
& +H_{20,1} w_{0} w_{1}+\frac{1}{2} H_{02,2} \beta_{2}^{2} \\
& +H_{21,0} \beta_{1} w_{0}+H_{21,1} \beta_{1} w_{1} \\
& +H_{12,0} \beta_{2} w_{0}+H_{12,1} \beta_{2} w_{1} \\
& +\mathcal{O}\left(\left|w_{0}\right|^{3}+\left|w_{0}^{2} w_{1}\right|+w_{1}^{2}\right) \\
& +\mathcal{O}\left(\beta_{1}^{2}+\left|\beta_{1} \beta_{2}\right|+\left|\beta_{2}\right|^{3}\right) .
\end{aligned}
$$

From linear and quadratic $w$-terms in the homological equation, one obtains

$$
\begin{aligned}
a & =\frac{1}{2} p_{1}^{T} B\left(q_{0}, q_{0}\right), \\
b & =p_{0}^{T} B\left(q_{0}, q_{0}\right)+p_{1}^{T} B\left(q_{0}, q_{1}\right)
\end{aligned}
$$

(see, for example [Kuznetsov, 2004]). Moreover,

$$
\begin{aligned}
& H_{20,0}=A^{I N V}\left(2 a q_{1}-B\left(q_{0}, q_{0}\right)\right), \\
& H_{20,1}=A^{I N V}\left(b q_{1}+H_{20,0}-B\left(q_{0}, q_{1}\right)\right) .
\end{aligned}
$$

Using the expressions for $a$ and $b$ it is easily verified that the arguments of $A^{I N V}$ are in the range of $A$. In (11) we define $x=A^{I N V} y$ by solving the non-singular bordered system

$$
\left(\begin{array}{cc}
A & p_{1} \\
q_{0}^{T} & 0
\end{array}\right)\left(\begin{array}{l}
x \\
s
\end{array}\right)=\left(\begin{array}{l}
y \\
0
\end{array}\right)
$$

Collecting the linear $\beta$ and $w \beta$-terms, the solvability condition applied to the resulting systems yields

$$
\begin{aligned}
& A H_{01}+J_{1} K_{1}=\left[q_{1}, 0\right], \\
& p_{1}^{T} B\left(q_{0}, H_{01}\right)+p_{1}^{T} A_{1}\left(q_{0}, K_{1}\right) \\
& \quad=\frac{1}{2}\left[p_{1}^{T} B\left(q_{1}, q_{1}\right), 0\right], \\
& p_{0}^{T} B\left(q_{0}, H_{01}\right)+p_{1}^{T} B\left(q_{1}, H_{01}\right) \\
& \quad+p_{0}^{T} A_{1}\left(q_{0}, K_{1}\right)+p_{1}^{T} A_{1}\left(q_{1}, K_{1}\right) \\
& \quad=\left[-p_{0}^{T} B\left(q_{1}, q_{1}\right)+3 p_{0}^{T} H_{20,1}, 1\right] .
\end{aligned}
$$

Taking (12), (13) and (14) together, one computes $K_{1}=\left[K_{1,0}, K_{1,1}\right]$ and $H_{01}=\left[H_{01,0}, H_{01,1}\right]$ by solving the $(n+2)$-dimensional system

$$
\begin{gathered}
\left(\begin{array}{cc}
A & J_{1} \\
p_{1}^{T} \mathrm{~B} q_{0} & p_{1}^{T} A_{1} q_{0} \\
p_{0}^{T} \mathrm{~B} q_{0}+p_{1}^{T} \mathrm{~B} q_{1} & p_{0}^{T} A_{1} q_{0}+p_{1}^{T} A_{1} q_{1}
\end{array}\right)\left(\begin{array}{c}
H_{01} \\
K_{1}
\end{array}\right) \\
=\left(\begin{array}{cc}
q_{1} & 0 \\
\frac{1}{2} p_{1}^{T} B\left(q_{1}, q_{1}\right) & 0 \\
-p_{0}^{T} B\left(q_{1}, q_{1}\right)+3 p_{0}^{T} H_{20,1} & 1
\end{array}\right),
\end{gathered}
$$

where the expressions with matrix $\mathrm{B}$ have natural interpretation. 
We note that the existence and uniqueness of the solution to (15) requires that the $(n+2) \times(n+2)$ matrix

$$
M=\left(\begin{array}{cc}
A & J_{1} \\
p_{1}^{T} \mathrm{~B} q_{0} & p_{1}^{T} A_{1} q_{0} \\
p_{0}^{T} \mathrm{~B} q_{0}+p_{1}^{T} \mathrm{~B} q_{1} & p_{0}^{T} A_{1} q_{0}+p_{1}^{T} A_{1} q_{1}
\end{array}\right)
$$

is non-singular. The left $(n+2) \times n$ block has full rank $n$ since the null vector $q_{0}$ of $A$ is not orthogonal to the row vectors in the $(2,1)$ and $(3,1)$ block entries of $M$ if $a \neq 0$ and $b \neq 0$. Since the right block column of $M$ contains parameter derivatives in all entries, the non-singularity of $M$ is the transversality condition for the BT point.

To prove that $K_{1}$ is non-singular we proceed by contradiction. Suppose that there exist real variables $\gamma$ and $\eta$ not both zero, such that $K_{1}\left(\begin{array}{l}\gamma \\ \eta\end{array}\right)=0$. Then

$$
M\left(\begin{array}{l}
\xi \\
0 \\
0
\end{array}\right)=\left(\begin{array}{c}
\gamma q_{1} \\
\frac{\gamma}{2} p_{1}^{T} B\left(q_{1}, q_{1}\right) \\
-\gamma p_{0}^{T} B\left(q_{1}, q_{1}\right)+3 \gamma p_{0}^{T} H_{20,1}+\eta
\end{array}\right)
$$

with $\xi \in \mathbb{R}^{n}$. This implies that $A \xi=\gamma q_{1}$, hence $0=p_{1}^{T} A \xi=\gamma p_{1}^{T} q_{1}=\gamma$. Hence

$$
M\left(\begin{array}{l}
\xi \\
0 \\
0
\end{array}\right)=\left(\begin{array}{l}
0 \\
0 \\
\eta
\end{array}\right)
$$

with $\eta \neq 0$. This, in turn, implies that $\xi=\mu q_{0}$ for a scalar $\mu \neq 0$. From the second block row in $M$ we then have $\mu p_{1}^{T} B\left(q_{0}, q_{0}\right)=0$, which contradicts $a \neq 0$.

The system (15) above corrects [Beyn, 1994; Beyn et al., 2002], where a wrong formula to compute $K_{1}$ is suggested based on the assumption that $K_{1}$ satisfies the equation (12) only.

Finally, one finds the quadratic coefficients as in [Beyn et al., 2002]

$$
\begin{aligned}
K_{2} & =-\left(p_{1}^{T} z\right) K_{1,0}, \\
H_{02,2} & =-A^{I N V}\left(z+J_{1} K_{2}\right),
\end{aligned}
$$

where

$$
\begin{aligned}
z & =B\left(H_{01,1}, H_{01,1}\right)+2 A_{1}\left(H_{01,1}, K_{1,1}\right) \\
& +J_{2}\left(K_{1,1}, K_{1,1}\right)
\end{aligned}
$$

as well as

$$
H_{12,0}=-A^{I N V}\left(B\left(q_{0}, H_{01,1}\right)+A_{1}\left(q_{0}, K_{1,1}\right)\right)
$$

\section{Homoclinic asymptotics in the $2 \mathrm{D}$ normal form}

We consider now the problem of finding an explicit asymptotic for the homoclinic orbit in the BT normal form (8) for small parameter values.

The first step of the construction is a singular rescaling (blowup transformation) which anticipates the cuspoidal shape of the phase portrait in the $\left(w_{0}, w_{1}\right)$-plane. We truncate $(8)$ at $\mathcal{O}\left(\|w\|^{3}+\|w\|^{2}\|\beta\|\right)$, and apply a rescaling

$$
\begin{aligned}
& w_{0}=\frac{\varepsilon^{2}}{a} u, \quad w_{1}=\frac{\varepsilon^{3}}{a} v, \\
& \beta_{1}=-\frac{4}{a} \varepsilon^{4}, \quad \beta_{2}=\frac{b}{a} \varepsilon^{2} \tau, \quad \varepsilon t=s .
\end{aligned}
$$

We obtain

$$
\left\{\begin{array}{l}
\dot{u}=v \\
\dot{v}=-4+u^{2}+\varepsilon \frac{b}{a} v(\tau+u)
\end{array}\right.
$$


where the dot now indicates the derivative with respect to $s$. For $\varepsilon=0$ the system is Hamiltonian.

The equilibria of $(21)$ are the saddle $(2,0)$ and the (degenerate) focus $(-2,0)$ for sufficiently small $\varepsilon>0$. The focus is stable if $a b>0$, unstable if $a b<0$. As noticed by Beyn [1994], a solution

$$
\left(\begin{array}{c}
u \\
v \\
\varepsilon \\
\tau
\end{array}\right) \in C^{1}(\mathbb{R}, \mathbb{R}) \times C^{1}(\mathbb{R}, \mathbb{R}) \times \mathbb{R} \times \mathbb{R}
$$

to (21), where $u$ and $v$ are such that the limits

$$
\lim _{s \rightarrow \pm \infty}\left(\begin{array}{l}
u(s) \\
v(s)
\end{array}\right) \text { and } \lim _{s \rightarrow \pm \infty}\left(\begin{array}{c}
\dot{u}(s) \\
\dot{v}(s)
\end{array}\right)
$$

exist, defines a homoclinic orbit to the saddle $(2,0)$ of $(21)$ for the corresponding values of $(\varepsilon, \tau)$. Following Beyn[1994], we fix the phase of the homoclinic solutions by requiring that

$$
v(0)=0
$$

i.e. that the homoclinic orbit always crosses the $u$-axis at $s=0$. For $\varepsilon=0$ and any $\tau$, the system (21) has the well-known homoclinic solution with $v_{0}(0)=0$, namely

$$
\left(\begin{array}{l}
u_{0}(s) \\
v_{0}(s)
\end{array}\right)=2\left(\begin{array}{c}
1-3 \operatorname{sech}^{2}(s) \\
6 \operatorname{sech}^{2}(s) \tanh (s)
\end{array}\right)
$$

Hence there exists a trivial branch $\left(u_{0}, v_{0}, 0, \tau\right)$ of homoclinic orbits in $(21)$. It has been shown in [Beyn, 1994] that

$$
\tau_{0}=\frac{10}{7}
$$

is a bifurcation point, at which a non-trivial smooth branch of homoclinic solutions of (21) emanates transversally. This branch can be parametrized by $\varepsilon$ and approximated by

$$
\left(\begin{array}{c}
u \\
v \\
\varepsilon \\
\tau
\end{array}\right)=\sum_{l=0}^{L} \varepsilon^{l}\left(\begin{array}{c}
u_{l} \\
v_{l} \\
0 \\
\tau_{l}
\end{array}\right)+\varepsilon\left(\begin{array}{l}
0 \\
0 \\
1 \\
0
\end{array}\right),
$$

where the integer $L$ defines the order of the approximation. Each $\left(u_{l}, v_{l}\right)$ with $l \leq 1$ satisfies a linear inhomogeneous system that can be obtained by inserting the approximation into (21) and collecting the $\varepsilon^{l}$-terms for $l=0,1,2,3, \ldots$.

The $\varepsilon^{0}$-terms yield the Hamiltonian system, while the $\varepsilon^{1}$-terms yield

$$
\left\{\begin{array}{l}
\dot{u}_{1}=v_{1} \\
\dot{v}_{1}=2 u_{0} u_{1}+\frac{b}{a} v_{0}\left(\tau_{0}+u_{0}\right)
\end{array}\right.
$$

A unique solution to $(26)$ with $\lim _{s \rightarrow \pm \infty}\left(u_{1}(s), v_{1}(s)\right)=(0,0)$ and satisfying $v_{1}(0)=0$ will provide the first-order correction to the Hamiltonian homoclinic orbit, which Beyn [1994] was unable to find explicitly. In our case, it is possible since (26) has a simpler (normalized) form.

To obtain such a solution we first make the observation that $\varphi_{1}(s)=v_{0}(s)$ solves the homogeneous problem $\ddot{u}_{1}=2 u_{0} u_{1}$. This enables us to find a second solution of the homogeneous problem by reducing it to a first order problem. This gives

$$
\varphi_{2}(s)=2 \cosh ^{2}(s)+5+\frac{15 s \sinh (s)}{\cosh ^{3}(s)}-\frac{15}{\cosh ^{2}(s)} .
$$

As we have two linearly independent solutions, it is now straightforward (although tedious) to solve the inhomogeneous problem $\ddot{u}_{1}=2 u_{0} u_{1}+\frac{b}{a} v_{0}\left(\tau_{0}+u_{0}\right)$ with arbitrary $\tau_{0}$. It is given by $u_{1}=\left(c_{1}-g\right) \varphi_{1}+\left(c_{2}+f\right) \varphi_{2}$ 
where $c_{1}$ and $c_{2}$ are yet undetermined integration constants and

$$
\begin{aligned}
f(s) & =\frac{b}{35 a} \frac{\sinh ^{3}(s)\left(7 \tau_{0}-10\right)}{\cosh ^{3}(s)} \\
& +\frac{3 b}{70 a} \frac{\sinh ^{3}(s)\left(7 \tau_{0}-10\right)}{\cosh ^{5}(s)}-\frac{9 b}{7 a} \frac{\sinh ^{3}(s)}{\cosh ^{7}(s)} \\
g(s) & =\frac{6 b}{7 a} \log (2 \cosh (s))+s \frac{b}{28 a} \frac{\sinh (s)\left(7 \tau_{0}-10\right)}{\cosh (s)} \\
& +\frac{b}{28 a} \frac{\left(-7 \tau_{0}+1\right)}{\cosh ^{2}(s)}+s \frac{b}{56 a} \frac{\sinh (s)\left(7 \tau_{0}-10\right)}{\cosh ^{3}(s)} \\
& +\frac{3 b}{56 a} \frac{\left(7 \tau_{0}+30\right)}{\cosh ^{4}(s)}+s \frac{3 b}{56 a} \frac{\sinh (s)\left(-7 \tau_{0}-20\right)}{\cosh ^{5}(s)} \\
& -\frac{45 b}{28 a}\left(\frac{1}{\cosh ^{6}(s)}-s \frac{\sinh (s)}{\cosh (s)}\right) \\
& -\frac{b}{a}\left(\frac{\tau_{0}}{8}+\frac{1}{28}+\frac{6}{7} \log 2\right),
\end{aligned}
$$

for which $f(0)=g(0)=0$ holds. Now we check the limits of $u_{1}(s)$ for $s \rightarrow \pm \infty$. First we see that $\lim _{s \rightarrow \pm \infty} g(s) \varphi_{1}(s)=\lim _{s \rightarrow \pm \infty} \varphi_{1}(s)=0$. So we focus on the other terms. We find $\lim _{s \rightarrow \pm \infty}\left(c_{2}+f(s)\right)=$ $c_{2} \pm \frac{b}{a} \frac{7 \tau_{0}-10}{35}$. Thus we recover $\tau_{0}=\frac{10}{7}$ together with $c_{2}=0$. The phase condition $v_{1}(0)=0$ gives $c_{1}=0$ so that

$$
\begin{aligned}
& u_{1}(s)=-\frac{72 b}{7 a} \frac{\sinh (s) \log (\cosh (s))}{\cosh ^{3}(s)}, \\
& v_{1}(s)=-\frac{72 b}{7 a} \frac{\sinh ^{2}(s)+\left(1-2 \sinh ^{2}(s)\right) \log (\cosh (s))}{\cosh ^{4}(s)} .
\end{aligned}
$$

Collecting the $\varepsilon^{2}$-terms leads to the linear inhomogeneous system

$$
\left\{\begin{array}{l}
\dot{u}_{2}=v_{2} \\
\dot{v}_{2}=2 u_{0} u_{2}+\frac{b}{a} v_{0}\left(\tau_{1}+u_{1}\right)+\frac{b}{a} v_{1}\left(\tau_{0}+u_{0}\right)+u_{1}^{2}
\end{array}\right.
$$

where the homogeneous part has the same form as in (26). Thus, the general solution to (28) can be written as $u_{2}=\left(c_{3}-g_{1}\right) \varphi_{1}+\left(c_{4}+f_{1}\right) \varphi_{2}$ and $v_{2}=\dot{u}_{2}$, where $c_{3}$ and $c_{4}$ are new integration constants, while $g_{1}$ and $f_{1}$ are certain functions satisfying $f_{1}(0)=g_{1}(0)=0$, which we have obtained explicitly but are too long to be displayed here. A unique solution to (28) with $\lim _{s \rightarrow \pm \infty}\left(u_{2}(s), v_{2}(s)\right)=(0,0)$ and satisfying $v_{2}(0)=0$ will provide the second-order correction to the Hamiltonian homoclinic orbit. The requirements $\lim _{s \rightarrow \pm \infty}\left(c_{4}+f_{1}(s)\right)=0$ imply $c_{4}=\frac{9 b^{2}}{196 a^{2}}$ and $\tau_{1}=0$, while the phase condition $v_{2}(0)=0$ gives $c_{3}=0$. The condition $\tau_{1}=0$ is equivalent to $\tau^{\prime}(0)=0$ established in [Beyn, 1994] using symmetry arguments.

At this point we can conclude that the tangent line to the homoclinic branch at the bifurcation point is given by

$$
\left(\begin{array}{c}
u \\
v \\
\varepsilon \\
\tau
\end{array}\right)=\left(\begin{array}{c}
u_{0} \\
v_{0} \\
0 \\
\tau_{0}
\end{array}\right)+\varepsilon\left(\begin{array}{c}
u_{1} \\
v_{1} \\
1 \\
0
\end{array}\right) .
$$

In Fig. 1(a) we have plotted the corresponding tangent predictor in the $(u, v)$-plane for several values of $\varepsilon$. It is remarkable, see also the close-up, that the orbits for $\varepsilon \neq 0$ approach the saddle along the "wrong" direction, making a "parasitic turn" when $s \rightarrow+\infty$ or $s \rightarrow-\infty$ (see Fig.1(b)). Indeed, the difference $2-\left(u_{0}(s)+\varepsilon u_{1}(s)\right)$ asymptotically behaves for $s \rightarrow \pm \infty$ as

$$
\frac{288}{7} \frac{b}{a} \varepsilon s e^{\mp 2 s}
$$




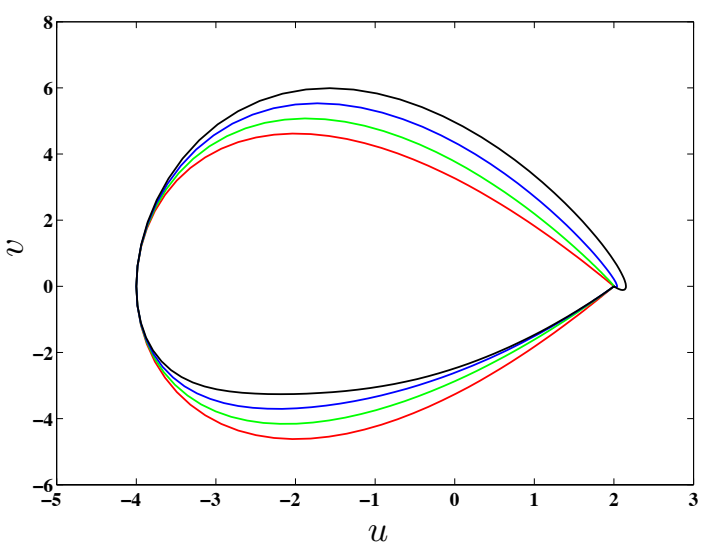

(a)

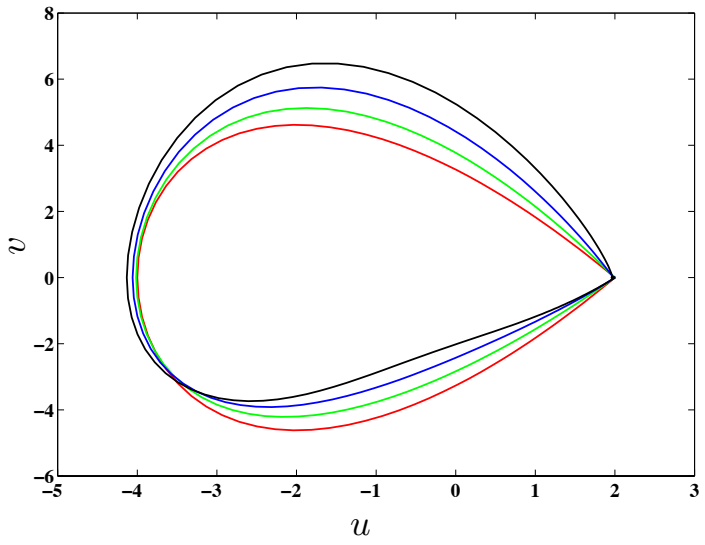

(c)

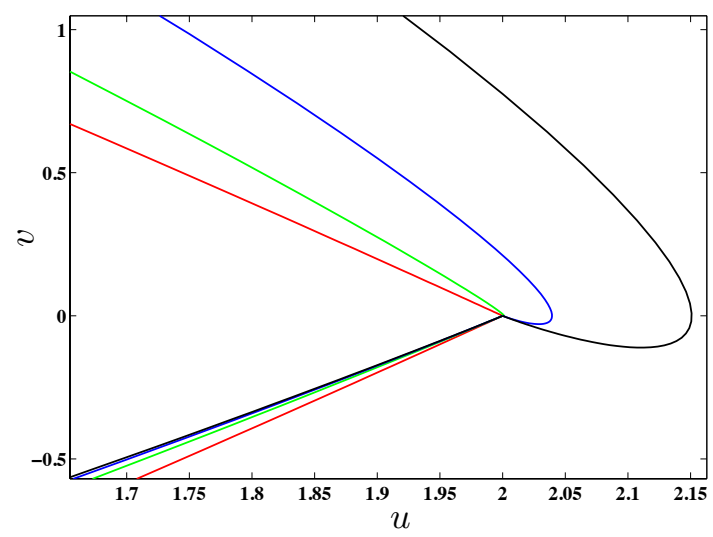

(b)

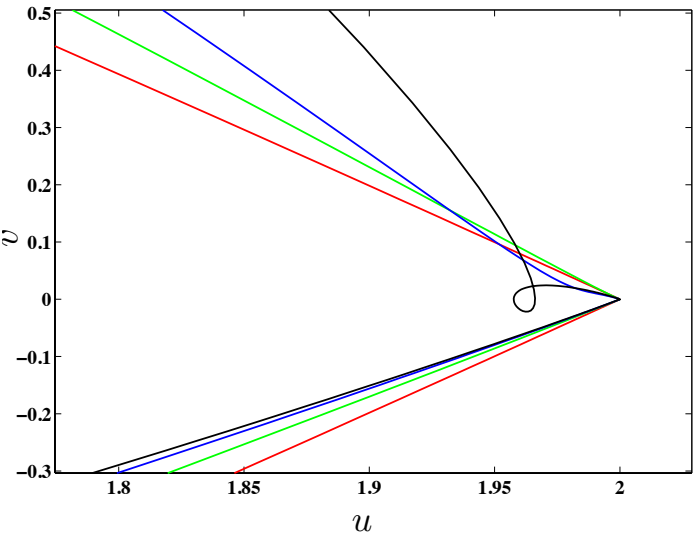

(d)

Fig. 1: Homoclinic orbit predictors for $(a, b)=(-1,1)$ and $\varepsilon=0$ (red), 0.2 (green), 0.4 (blue), 0.6 (black). (a) Tangent predictors; (b) Zoom of the tangent predictors near the saddle: the "parasitic turn" is clearly visible; (c) The second-order predictors; (d) Zoom of the second-order predictors near the saddle: the "parasitic loop" is present only for large $\varepsilon$.

and, therefore, is negative for $s \rightarrow-\infty$ if $a b>0$ or for $s \rightarrow+\infty$ if $a b<0$ (provided that $\varepsilon>0$ ). When $\varepsilon \rightarrow 0$, this parasitic turn vanishes and on any finite time interval $[-T, T]$ the tangent predictor with sufficiently small $\varepsilon$ does approximate the "true" homoclinic solution better than the Hamiltonian predictor with $\varepsilon=0$. In particular, while the Hamiltonian homoclinic orbit (23) is symmetric w.r.t. the $u$-axis, the tangent predictor defines a non-symmetric approximate orbit, better corresponding to the non-symmetric true homoclinic orbit in (21) and in the normal form (8), see Section 5.1 for a graphical illustration. 
With the found above constants $c_{3}, c_{4}$, and $\tau_{1}$, one obtains

$$
\begin{aligned}
u_{2} & =-\frac{216 b^{2}}{49 a^{2}} \frac{\log ^{2}(\cosh (t))(\cosh (2 t)-2)}{\cosh ^{4}(t)} \\
& -\frac{216 b^{2}}{49 a^{2}} \frac{\log (\cosh (t))(1-\cosh (2 t))}{\cosh ^{4}(t)} \\
& -\frac{18 b^{2}}{49 a^{2}} \frac{(6 t \sinh (2 t)-7 \cosh (2 t)+8)}{\cosh ^{4}(t)}, \\
v_{2} & =\frac{216 b^{2}}{49 a^{2}} \frac{t\left(2 \cosh ^{2}(t)-3\right)}{\left.\cosh ^{4}(t)\right)} \\
& +\frac{288 b^{2}}{49 a^{2}} \frac{\sinh (t)\left(3 \log ^{2}(\cosh (t))-6 \log (\cosh (t))\right)}{\cosh ^{3}(t)} \\
& -\frac{216 b^{2}}{49 a^{2}} \frac{\sinh (t)\left(12 \log ^{2}\left(\cosh ^{2}(t)\right)-14 \log (\cosh (t))\right)}{\cosh ^{5}(t)} \\
& -\frac{288 b^{2}}{49 a^{2}} \frac{\sinh ^{2}(t)}{\cosh ^{3}(t)}+\frac{648 b^{2}}{49 a^{2}} \frac{\sinh ^{2}(t)}{\cosh ^{5}(t)} .
\end{aligned}
$$

Figs. 1(c) and (d) show the second-order predictor in the $(u, v)$-plane. While no "parasitic turn" exists for small $\varepsilon$, a "parasitic loop" appears for big values of $\varepsilon$. Thus implies that in actual computations $\varepsilon$ must be small, as one naturally expects for asymptotic expansions.

Since we need the value of $\tau_{2}$ in the next section, we have to make one more step. Collecting the $\varepsilon^{3}$-terms leads to the linear inhomogeneous system

$$
\left\{\begin{aligned}
\dot{u}_{3} & =v_{3}, \\
\dot{v}_{3} & =2 u_{0} u_{3}+\frac{b}{a} v_{0}\left(\tau_{2}+u_{2}\right)+\frac{b}{a} v_{1}\left(\tau_{2}+u_{2}\right) \\
& +\frac{b}{a} v_{2}\left(\tau_{0}+u_{0}\right)+2 u_{1} u_{2},
\end{aligned}\right.
$$

As before, $u_{3}=\left(c_{5}-g_{2}\right) \varphi_{1}+\left(c_{6}+f_{2}\right) \varphi_{2}$ for some functions $g_{2}$ and $f_{2}$ satisfying $f_{2}(0)=g_{2}(0)=0$. The conditions $\lim _{s \rightarrow \pm \infty} u_{3}(s)=0$ imply, in particular,

$$
\tau_{2}=\frac{288 b^{2}}{2401 a^{2}}
$$

Thus, the second-order approximation for the emanating homoclinic orbits for the BT normal form (8) is given by

$$
\begin{aligned}
w_{0}(t) & =\frac{\varepsilon^{2}}{a}\left(u_{0}(\varepsilon t)+\varepsilon u_{1}(\varepsilon t)+\varepsilon^{2} u_{2}(\varepsilon t)\right) \\
& +\mathcal{O}\left(\varepsilon^{5}\right), \\
w_{1}(t) & =\frac{\varepsilon^{3}}{a}\left(v_{0}(\varepsilon t)+\varepsilon v_{1}(\varepsilon t)+\varepsilon^{2} v_{2}(\varepsilon t)\right) \\
& +\mathcal{O}\left(\varepsilon^{6}\right), \\
\beta_{1} & =-\varepsilon^{4}\left(\frac{4}{a}\right)+\mathcal{O}\left(\varepsilon^{5}\right), \\
\beta_{2} & =\varepsilon^{2} \tau_{0}\left(\frac{b}{a}\right)+\varepsilon^{4} \tau_{2}\left(\frac{b}{a}\right)+\mathcal{O}\left(\varepsilon^{5}\right),
\end{aligned}
$$

where all ingredients are defined above.

\section{Computational formulas for $n$-dimensional systems}

In this section we provide an asymptotic expression for the bifurcating homoclinic solution of (1). To find the prediction, we transfer system (8) with our homoclinic approximation (32) back to the original form 
(1). With data collected in (10) and (11), and using (15) and (17)-(19), we get the second-order homoclinic predictor for the original system (1)

$$
\begin{aligned}
\alpha & =\varepsilon^{2} \frac{10 b}{7 a} K_{1,1} \\
& +\varepsilon^{4}\left(-\frac{4}{a} K_{1,0}+\frac{50 b^{2}}{49 a^{2}} K_{2}+\frac{288 b^{3}}{2401 a^{3}} K_{1,1}\right) \\
& +\mathcal{O}\left(\varepsilon^{5}\right)
\end{aligned}
$$

and

$$
\begin{aligned}
x(t)= & \varepsilon^{2}\left(\frac{10 b}{7 a} H_{01,1}+\frac{1}{a} u_{0}(\varepsilon t) q_{0}\right) \\
+ & \varepsilon^{3}\left(\frac{1}{a} v_{0}(\varepsilon t) q_{1}+\frac{1}{a} u_{1}(\varepsilon t) q_{0}\right) \\
+ & \varepsilon^{4}\left(-\frac{4}{a} H_{01,0}+\frac{50 b^{2}}{49 a^{2}} H_{02,2}+\frac{288 b^{3}}{2401 a^{3}} H_{01,1}\right. \\
& +\frac{1}{a} u_{2}(\varepsilon t) q_{0}+\frac{1}{a} v_{1}(\varepsilon t) q_{1} \\
& \left.+\frac{1}{2 a^{2}} H_{20,0} u_{0}(\varepsilon t)^{2}+\frac{10 b}{7 a^{2}} H_{12,0} u_{0}(\varepsilon t)\right) \\
+ & \mathcal{O}\left(\varepsilon^{5}\right) .
\end{aligned}
$$

We note that the phase condition (22) could be replaced by a different one. We can fix the phase using an integral condition as in [Doedel \& Kernévez, 1986; Champneys et al., 1996] and [De Witte et al., 2012]. Namely, we can fix the phase of $u(s)$ by requiring its minimal $L_{2}$-distance to the Hamiltonian approximation $u_{0}(s)$. Mathematically, this leads to $\int_{-\infty}^{+\infty}\left\langle u(s)-u_{0}(s), \dot{u}_{0}(s)\right\rangle d s=0$ where $\dot{u}_{0}(s)=v_{0}(s)$. Applied for $u_{1}$ and $u_{2}$ separately, this gives different values of $c_{1}$ and $c_{4}$. However, extensive numerical tests did not show any substantial superiority of this choice over the predictor based on (33) and (34).

\section{Examples}

\subsection{Truncated normal form}

Consider the two-parameter system

$$
\left\{\begin{array}{l}
\dot{w}_{0}=w_{1} \\
\dot{w}_{1}=\beta_{1}+\beta_{2} w_{1}+a w_{0}^{2}+b w_{0} w_{1}
\end{array}\right.
$$

that is the truncated normal form (8). Bifurcation analysis of this system has been presented, for example, in [Guckenheimer \& Holmes, 1983]. In this system we have two equilibria $\left( \pm \sqrt{-\frac{1}{a} \beta_{1}}, 0\right)$, which lie on the folded surface $S=\left\{\left(w_{0}, w_{1}, \beta_{1}, \beta_{2}\right)=\left(s, 0,-a s^{2}, \beta_{2}\right): s, \beta_{2} \in \mathbb{R}\right\}$. We want to compare the predicted homoclinic solution with that obtained via the high-accuracy numerical continuation in MatCont [De Witte et al., 2012].

Substituting $\tau=\tau_{0}+\tau_{2} \varepsilon^{2}$ with (24) and (31) into (20), we obtain the following second-order approximation for the homoclinic bifurcation curve in the parameter plane $\left(\beta_{1}, \beta_{2}\right)$ of $(35)$ :

$$
\beta_{2}=-\frac{72 b^{3}}{2401 a^{2}} \beta_{1}+\frac{5 b}{7 a} \sqrt{-a \beta_{1}}
$$

for $\operatorname{sign}\left(\beta_{1}\right)=-\operatorname{sign}(a)$. For $a=-1$ and $b=1$, this approximation is shown as the red curve Hom ${ }^{\text {Pred }}$ in Fig.2a.

We use MatCont to start a continuation of equilibria with initial parameter values $\left(\beta_{1}, \beta_{2}\right)=(1,-2)$ and the equilibrium point $\left(w_{0}, w_{1}\right)=(1,0) ; \beta_{2}$ is the free parameter. Two bifurcation points are detected along the curve of equilibria, limit point $(\mathrm{LP})$ and Hopf $(\mathrm{H})$. The LP continuation is now carried out 


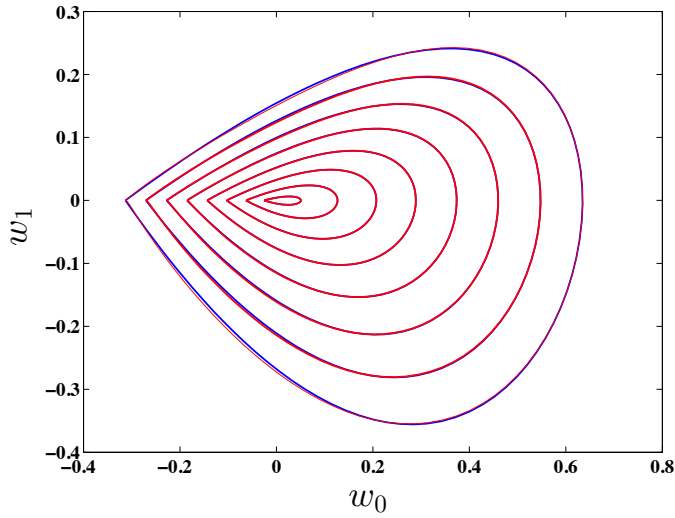

(a)

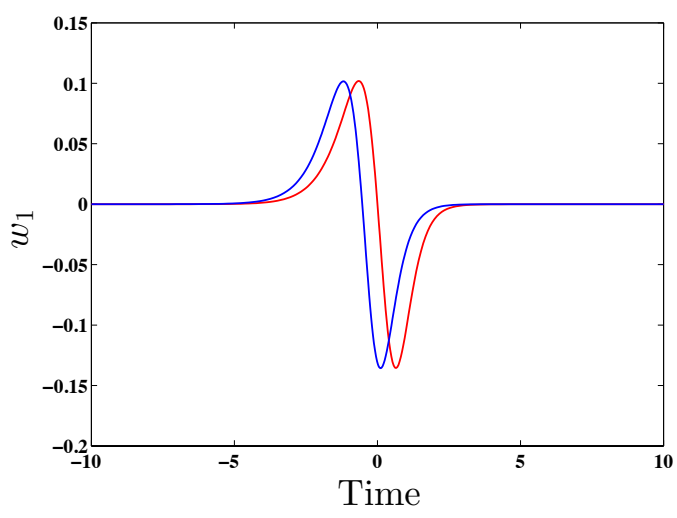

(c)

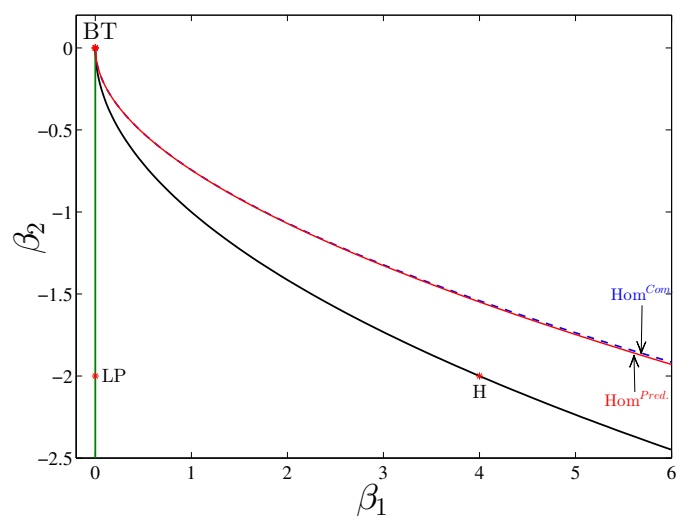

(b)

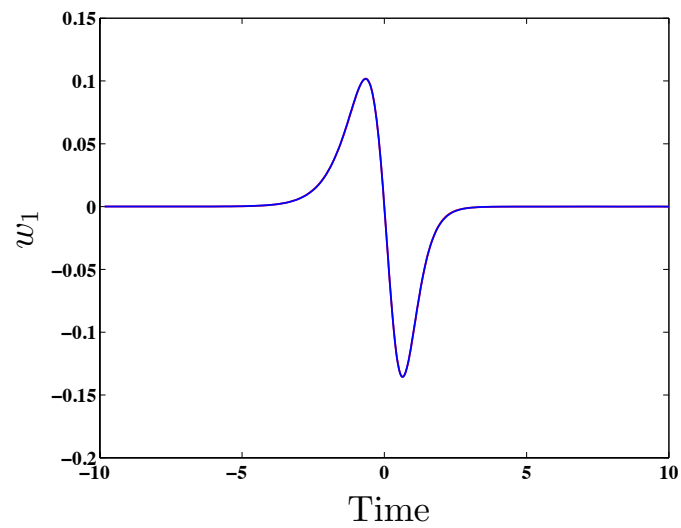

(d)

Fig. 2: (a) The comparison of homoclinic orbits in phase space between computed (blue) and predicted (red) using the second-order correction for $\log 10\left(\beta_{1}\right)=-3.921,-3.467,-2.603,-2.094,-1.772,-1.532$, $-1.342,-1.183,-1.049$. (b) Predicted (red) and computed (dashed blue) homoclinic bifurcation curves in parameter space are hardly distinguishable. The green line is the LP curve. The black curve is the Hopf curve. (c) and (d) The time shift so that the predicted (red) and computed (blue) curves consider at $t=0$ for $\log 10\left(\beta_{1}\right)=-1.532$.

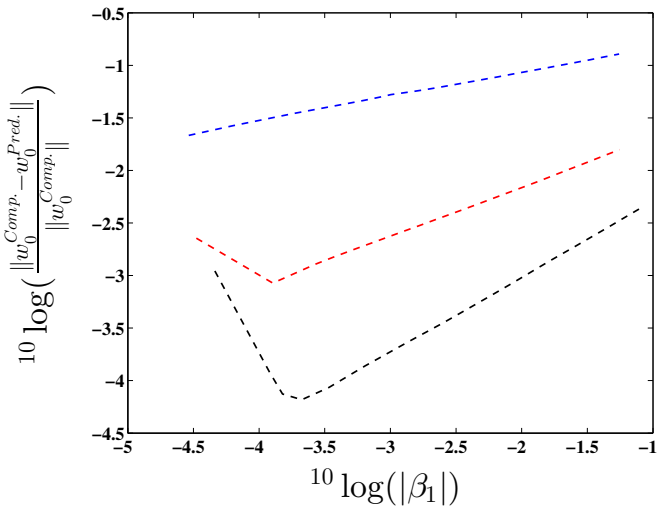

(a)

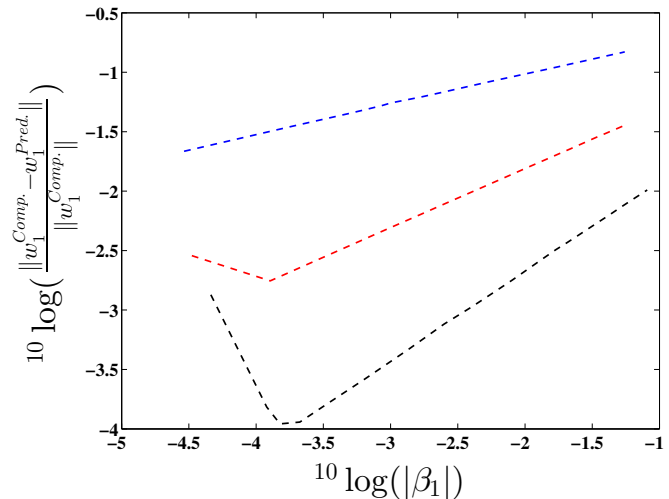

(b)

Fig. 3: Accuracy of the predicted $w_{0}$ and $w_{1}$ functions with Hamiltonian solution (blue), tangent predictor (red) and second-order predictor (black) for $\beta_{1} \in\left[3.85 \times 10^{-5}, 0.05\right]$. This corresponds to $\varepsilon \in[0.05,0.35]$. Euclidean norms of the vectors of the function values in the points of the fine mesh are used. 
to detect the BT-bifurcation point at $\left(\beta_{1}, \beta_{2}\right)=(0,0)$. We start the homoclinic continuation using the BT_Hom initializer, using the predictor (33)-(34) with $\varepsilon=0.08$. This yields the dashed blue curve Hom ${ }^{C o m}$ in Fig. 2a. In Fig. 2b we show the corresponding homoclinic orbits. Observe that the homoclinic orbit is indeed non-symmetric w.r.t. $w_{0}$, as is correctly predicted by the improved starter. Next in Fig. 3, we compare the relative errors of the orbit as a function of $\beta_{1}$. The error is computed by taking a computed homoclinic orbit from the numerical continuation. This gives a value of $\beta_{1}$ which yields a value for $\varepsilon$ in the predictor. The predictors are then compared with the computed solution using the time points of the fine mesh in the numerical continuation, after a time shift so that the computed and predicted curves coincide for $t=0$. We see that the improved predictors (red,black) perform better than the one based on the Hamiltonian solution (blue).

\subsection{Indirect field oriented control system}

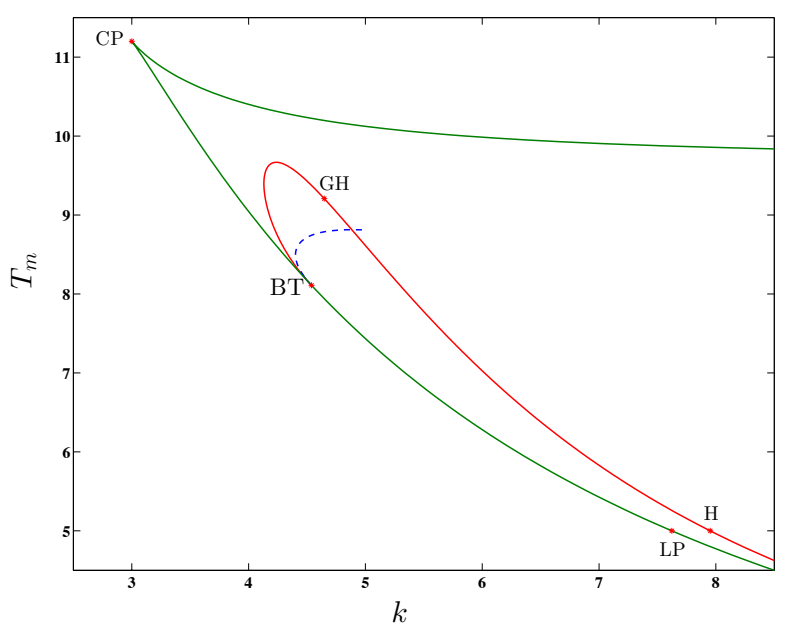

(a)

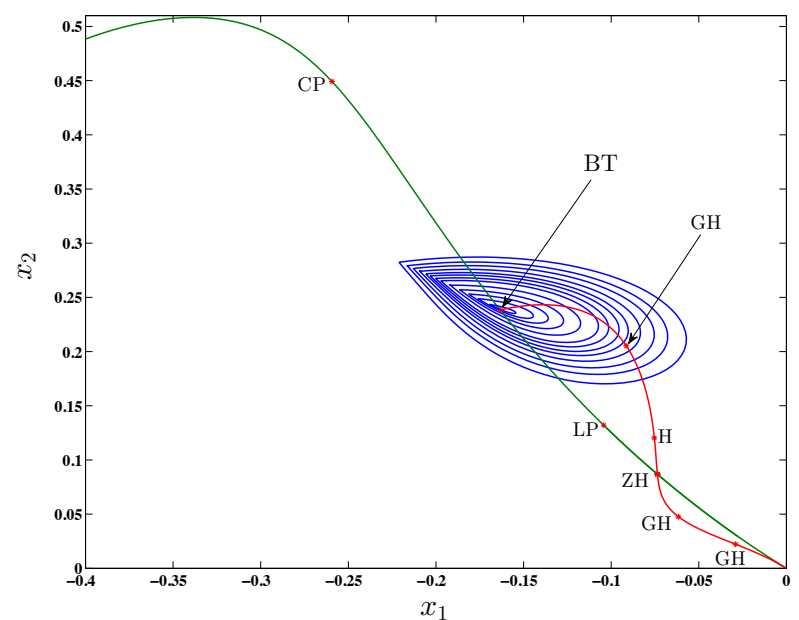

(b)

Fig. 4: (a) Homoclinic orbits emanating from the BT point of the IFOC model in parameter space $\left(k, T_{m}\right)$. The dashed blue curve is the homoclinic orbit. The red line is the Hopf curve and the green line is the LP curve. (b) The homoclinic orbits in state space $\left(x_{1}, x_{2}\right)$. The green line is the LP curve with three codim 2 points. The red line is the Hopf curve with three generalized Hopf bifurcation points $(\mathrm{GH})$ and $\mathrm{ZH}$ point.

The indirect field oriented control (IFOC) system of an induction motor can be mathematically described as in [Bazanella \& Reginatto, 2000] and [Salas et al., 2008] by the following ODEs:

$$
\left\{\begin{aligned}
\dot{x}_{1} & =-c_{1} x_{1}+c_{2} x_{4}-\frac{k c_{1}}{u_{2}^{0}} x_{2} x_{4}, \\
\dot{x}_{2} & =-c_{1} x_{2}+c_{2} u_{2}^{0}+\frac{k c_{1}}{u_{2}^{0}} x_{1} x_{4}, \\
\dot{x}_{3} & =-c_{3} x_{3}-c_{4} c_{5}\left(x_{2} x_{4}-u_{2}^{0} x_{1}\right) \\
& +c_{4}\left(T_{m}+\frac{c_{3}}{c_{4}} w_{r e f}\right), \\
\dot{x}_{4} & =-\left(k_{i}-k_{p} c_{3}\right) x_{3}-k_{p} c_{4} c_{5}\left(x_{2} x_{4}-u_{2}^{0} x_{1}\right) \\
& +k_{p} c_{4}\left(T_{m}+\frac{c_{3}}{c_{4}} w_{r e f}\right) .
\end{aligned}\right.
$$

Here $x_{1}, x_{2}, x_{3}$ and $x_{4}$ are the state variables, where $x_{1}$ and $x_{2}$ denote direct and quadrature components of the rotor flux; $x_{3}$ is the rotor speed error (i.e., the difference between the reference and the real mechanical speeds of the rotor); and $x_{4}$ denotes the quadrature axis component of the stator current. We also define the following constants and parameters: $u_{2}^{0}$ is a constant reference for the rotor flux magnitude; $c_{1}$ to $c_{5}$ are machine parameters; $k_{p}$ and $k_{i}$ are the proportional and the integral PI control gains, respectively; $w_{\text {ref }}$ is the speed reference; $T_{m}$ the load torque; $k$ the measure of rotor time constant mismatches. The 
occurrence of LP and Hopf in IFOC have been characterized as a result of rotor time constant mismatches (see, for example, [Bazanella \& Reginatto, 2002; Gordillo et al., 2002] and [Moiola \& Chen, 1996]). The first results on the occurrence of a BT bifurcation in the IFOC model were presented in [Salas et al., 2004]. A detailed analytical study for the codim 2 bifurcations of (37) can be found in [Salas et al., 2008]. In this paper, we shall examine only the homoclinic orbits that emanate from a given BT point. By continuation of equilibria starting with parameters $k=17, T_{m}=5, c_{1}=4.4868, c_{2}=0.3567, c_{3}=0, c_{4}=9.743$, $c_{5}=1.911, u_{2}^{0}=11.3, k_{p}=4.5, k_{i}=500, w_{r e f}=0$, and initial point $\left(x_{1}, x_{2}, x_{3}, x_{4}\right)=(-0.21,0.11,2.5)$, MatCont detects an LP point and a Hopf point. Further, by continuation of the limit point with $\left(k, T_{m}\right)$ free, three codim 2 points are detected, BT, ZH and CP.

Table 1: Parameter and state values at the bifurcation points in Fig.4.

\begin{tabular}{cccc}
\hline Label & $k$ & $T_{m}$ & State variables \\
\hline \hline BT & 04.54 & 08.12 & $(-0.16,0.24,0.00,10.06)$ \\
CP & 03.00 & 11.20 & $(-0.26,0.45,0.00,06.52)$ \\
ZH & 11.21 & 03.43 & $(-0.07,0.09,0.00,11.12)$ \\
\hline
\end{tabular}

The normal form coefficients for the BT point are $(a, b)=(28.01,-0.91)$. From the BT point we start the continuation of the homoclinic curve, using $k$ and $T_{m}$ as free system parameters and with initial amplitude value $\varepsilon=0.008$ (see Fig.4). This shows that our improved predictor works in higher dimensional systems as well.

\section{References}

Arnold, V. I. [1983] Geometrical Methods in the Theory of Ordinary Differential Equations (SpringerVerlag, New York, Heidelberg, Berlin).

Bazanella, A. S. \& Reginatto, R. [2000] "Robustness margins for indirect field oriented control of induction motors," IEEE Trans. Autom. Contr. 45, 1226-1231.

Bazanella, A. S. \& Reginatto, R. [2002] "Instabilty mechanisms in indirect field oriented control drives: Theory and experimental results," IFAC World Congress (Barcelona, Spain), Vol. 15, Part I.

Beyn, W.-J. [1994] "Numerical analysis of homoclinic orbits emanating from a Takens-Bogdanov point," IMA J. Numer. Anal. 14, 381-410.

Beyn, W.-J., Champneys, A., Doedel, E. J., Govaerts, W., Kuznetsov, Yu. A. \& Sandstede, B. [2002] "Numerical Continuation, and Computation of Normal Forms," Handbook of Dynamical Systems, II, ed. Fiedler, B. (Elsevier Science, North Holland), pp. 149-219.

Bogdanov, R. I. [1975] "Versal deformations of a singular point on the plane in the case of zero eigenvalues," Functional Anal. Appl. 9, 144-145.

Bogdanov, R. I. [1976a] "Bifurcations of a limit cycle of a certain family of vector fields on the plane," Proceedings of Petrovskii Seminar, Vol. 2 (Moscow State University, Moscow), pp. 23-35, in Russian (English translation: Selecta Math. Soviet. 1, 1981, 373-388).

Bogdanov, R. I. [1976b] "The versal deformation of a singular point of a vector field on the plane in the case of zero eigenvalues," Proceedings of Petrovskii Seminar, Vol. 2 (Moscow State University, Moscow), pp. 37-65, in Russian (English translation: Selecta Math. Soviet. 1, 1981, 389-421).

Champneys, A. R., Kuznetsov, Yu. A. \& Sandstede, B. [1996] "A numerical toolbox for homoclinic bifurcation analysis," Internat. J. Bifur. Chaos Appl. Sci. Engrg. 6, 867-887.

De Witte, V., Govaerts, W., Kuznetsov, Yu. A. \& Friedman, M. [2012] "Interactive initialization and continuation of homoclinic and heteroclinic orbits in MATLAB," ACM Trans. Math. Software 38, Art. 18, 34 .

Dhooge, A., Govaerts, W. \& Kuznetsov, Yu. A. [2003] "MATCONT: a MATLAB package for numerical bifurcation analysis of ODEs," ACM Trans. Math. Software 29, 141-164.

Dhooge, A., Govaerts, W., Kuznetsov, Yu. A., Meijer, H. G. E. \& Sautois, B. [2008] "New features of the 
software MatCont for bifurcation analysis of dynamical systems," Math. Comput. Model. Dyn. Syst. 14, 147-175.

Doedel, E. J. \& Kernévez, J. P. [1986] "Auto: Software for continuation and bifurcation problems in ordinary differential equations," Applied mathematics report, California Institute of Technology, Pasadena CA, 226 pages.

Gordillo, F., Salas, F., Ortega, R. \& Aracil, J. [2002] "Hopf bifurcation in indirect field-oriented control of induction motors," Automatica 38, 829-835.

Govaerts, W. J. F. [2000] Numerical methods for bifurcations of dynamical equilibria (SIAM, Philadelphia, $\mathrm{PA})$.

Guckenheimer, J. \& Holmes, P. [1983] Nonlinear Oscillations, Dynamical Systems and Bifurcations of Vector Fields (Springer-Verlag, New York).

Kuznetsov, Yu. A. [2004] Elements of Applied Bifurcation Theory, 3rd ed. (Springer-Verlag, New York).

Moiola, J. \& Chen, G. [1996] Hopf Bifurcation Analysis: A Frequency Domain Approach (World Scientific, Singapore).

Salas, F., Gordillo, F. \& Aracil, J. [2008] "Codimension-two bifurcations in indirect field oriented control of induction motor drives," Internat. J. Bifur. Chaos 18, 779-792.

Salas, F., Reginatto, R., Gordillo, F. \& Aracil, J. [2004] "Bogdanov-Takens bifurcation in indirect field oriented control of induction motor drives," Proc. CDC (Atlantis, Bahamas), pp. 4357-4362.

Takens, F. [1974] "Forced oscillations and bifurcations," Comm. Math. Inst., Rijksuniversiteit Utrecht 2, 1-111, reprinted in Global Analysis of Dynamical Systems, Instute of Physics, Bristol, 2001, pp. 1-61. 